С.К. Удовиченко ${ }^{1}$, К.В. Жуков ${ }^{1}$, А.В. Топорков ${ }^{1}$, Д.В. Викторов ${ }^{1}$, О.В. Зубарева ${ }^{2}$, И.А. Климина ${ }^{2}$, М.Н. Таратутина ${ }^{2}$ Ю.В. Кетов ${ }^{2}$

\title{
Эпидемические проявления COVID-19 на территории
}

\section{Волгоградской области}

${ }^{1}$ ФКУЗ «Волгоградский научно-исследовательский противочумный институт» Роспотребнадзора, Волгоград, Россия

${ }^{2}$ Управление Роспотребнадзора по Волгоградской области, Волгоград, Россия

Проведена оценка проявлений эпидемического процесса COVID-19 в Волгоградской области по интенсивности, территориальному распространению, среди различных групп населения (пол, возраст, социальный статус), особенностям клинической форм заболевания. Показано, что отмечается стабильное снижение заболеваемости новой коронавирусной инфекций, проявляющееся сокращением общей и групповой заболеваемости, количества семейных очагов инфекции, числа госпитализированных пациентов. В структуре заболевших наблюдается преобладание лиц женского пола (54,2 \%), что сопоставимо с данными по Российской Федерации. Наибольший процент заболевших приходится на возрастные группы 40-49 лет (18,1 \%) и 50-59 лет (19,8 \%). В клинической картине заболевания преобладают легкие и бессимптомные формы, отмечаемые в примерно равном соотношении (35,4 и 34,5 \% соответственно). В Волгоградской области установлена более низкая частота регистрации тяжелых форм и летальных исходов по сравнению с Российский Федерацией в целом. В социальной структуре заболевших наибольшую долю составляют пенсионеры (25,2 \%) и рабочие $(11,7 \%)$.

Ключевые слова: COVID-19, эпидемический процесс, структура заболеваемости, Волгоградская область.

31 декабря 2019 г. Китайская Народная Республика проинформировала В03 о появлении ранее неизвестной инфекционной болезни, протекавшей в виде кластера пневмоний в г. Ухань [10]. Быстро распространившись в начале в масштабе эпидемии в Китае, уже через месяц болезнь диагностирована в 18 странах мира, на основании чего данная ситуация верифицирована ВОЗ как чрезвычайная ситуация в области общественного 
здравоохранения международного значения [2]. 11 марта 2020 г. ВОЗ объявила стремительное распространение новой коронавирусной инфекции COVID-19 пандемией, впервые присвоив эпидемической ситуации глобальный статус после пандемии гриппа А (H1N1) в 2009 г. [1]. К настоящему времени (на 24.07.2020 г.) болезнь охватила более 200 стран и территорий на всех континентах с регистрацией 15,6 млн случаев заболеваний и 637,8 тыс. смертельных исходов, вызвав рекордно высокие тяжелые социально-экономические последствия, нарушение массовой общественной и производственной деятельности людей, и потребовавшей необходимости организации и координации мер борьбы на уровне высших должностных лиц государств.

В Российской Федерации случаи заболевания COVID-19 регистрируются с 31 января 2020 г. В настоящее время (по данным на 24.07.2020 г.) лабораторно подтверждено 789771 случай инфицирования SARS-Cov-2 во всех субъектах страны, включая 12784 смертельных исхода. С учетом появляющихся в открытой печати работ [3, 4], посвящённых всесторонней оценке эпидемиологической ситуации по COVID-19 в Российской Федерации, представляется актуальным проанализировать особенности течения эпидемического процесса на примере отдельного субъекта страны (Волгоградской области).

Первый случай заболевания в Волгоградской области лабораторно подтвержден 24.03.2020 г. у жительницы г. Волгограда, возвратившейся из ОАЭ 16.03.2020 г. В последующем до конца марта 2020 г. регистрировались спорадические случаи инфицирования SARS-Cov-2, а, начиная со второй недели апреля, динамика заболеваемости характеризовалась ростом ежедневно регистрируемых случаев. Пиковые значения заболеваемости достигнуты 1 июня (189 новых заболеваний), в то время как максимум заболеваемости в Москве и Московской области пришелся практически на месяц раньше (6 мая - 7446 случаев), а в остальной части России - на 10 дней раньше (21 мая - 6021 случай). С 23 недели (01.06.-07.06.) в регионе 
отмечается стабильное снижение заболеваемости новой коронавирусной инфекций COVID-19, темп прироста за последние 7 недель (с 23 по 29 неделю) уменьшился с 23,6 \% до 7,2 \% (рис. 1).

На фоне еженедельного стабильного снижения общей заболеваемости COVID-19 наблюдается снижение групповой заболеваемости в организованных коллективах (с 11,6 \% на 23 неделе до 7,4 \% на 28 неделе и 1,3 \% на 29 неделе), количества домашних очагов и лиц, заболевших по контакту в домашних очагах (с 788 на 23 неделе до 548 на 29 неделе). Показатель очаговости на 23 неделе составлял 2,2, на 29 неделе - 1,4.

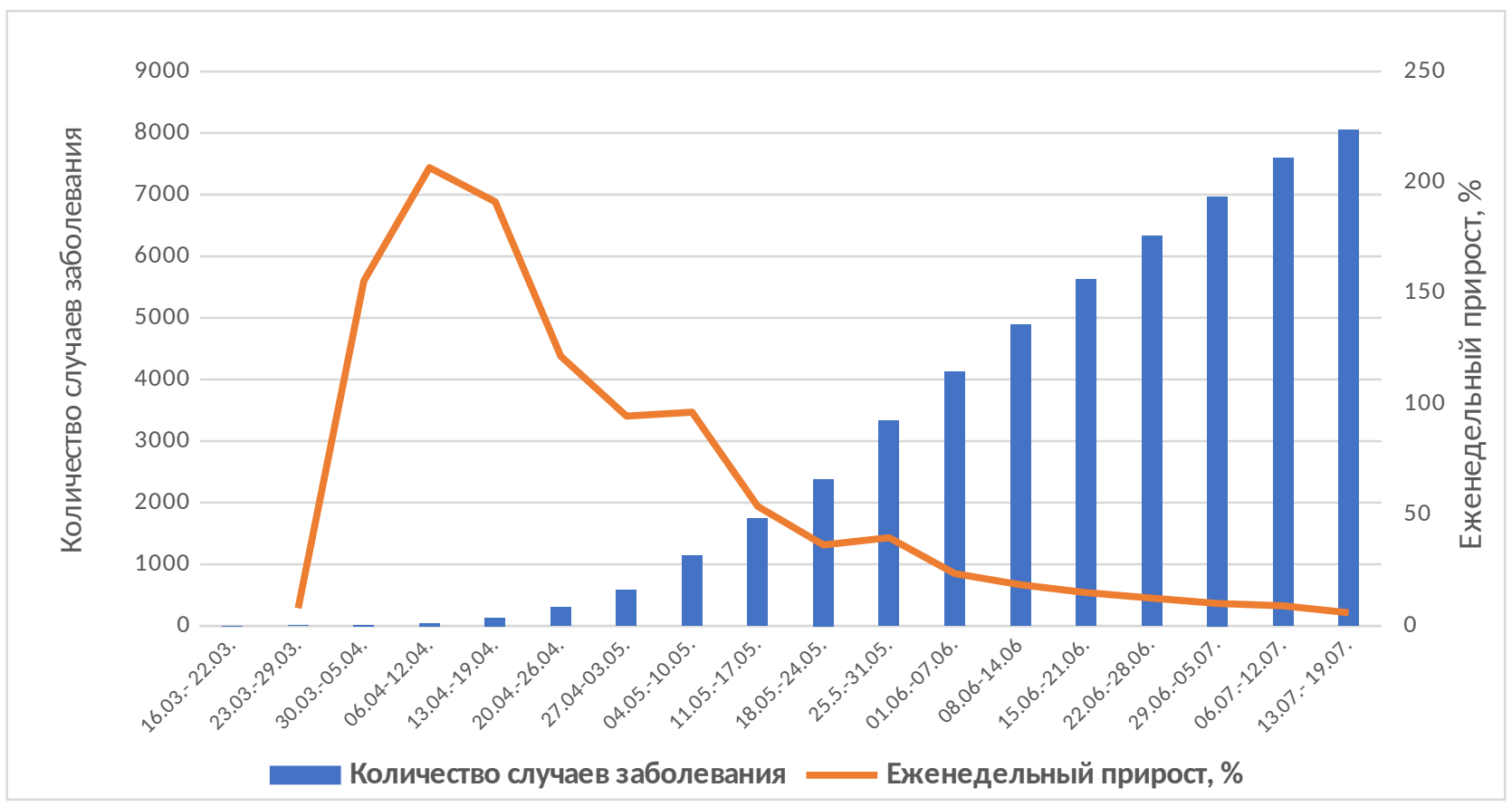

Рис. 1. Динамика заболеваемости COVID-19 в Волгоградской области

Об улучшении эпидемиологической обстановки в регионе может также свидетельствовать отмечаемая тенденция к снижению доли госпитализированных лиц. По состоянию на 22.05 .2020 г. доля госпитализированных лиц составляла 94 \%, на 20.07. - 57,6 \%. За последние три недели уровень госпитализации остается стабильным на уровне 16,7-17,3 на 10 тыс. населения. Следует отметить увеличение доли выздоровевших пациентов с 31,3 \% до 56,7 \%. 
Всего за период с 24.03.2020 г. по 24.07.2020 г. на территории Волгоградской области выявлено 8517 случаев инфицирования SARS-Cov-2, показатель заболеваемости составил 341,9 на 100 тысяч населения. На долю региона пришлось 1,06 \% от всех случаев заболеваний COVID-19 в России.

Анализ данных по заболеваемости различных половозрастных групп населения Волгоградской области показал, что заболеваемость среди мужчин составляет 45,8 \%, женщин - 54,2 \%. Эти данные сопоставимы с данными по Российской Федерации [3], в то время как в мире большинство пациентов составляют мужчины (50-75 \%) [5, 8]. В возрасте 0-18 лет достоверных различий в распределении заболеваемости по полу не выявлено, в группе от 18 до 30 лет среди заболевших превалировали лица мужского пола, от 30 лет и старше - женского пола. Следует отметить, что в целом по Российской Федерации более высокая частота регистрации случаев заболевания среди женщин отмечалась в возрастной группе 40 лет и старше, что обусловлено превалированием женского пола с этого возраста в популяции [4].

Случаи заболевания отмечены во всех возрастных группах. Наибольшее количество заболевших приходится на контингент лиц в возрасте 40-49 лет (18,1 \%) и 50-59 лет (19,8 \%), т.е. активного и трудоспособного населения. О сходном распределении случаев заболеваний COVID-19 по возрастным группам сообщалось в зарубежных публикациях [8]. Среди лиц в возрасте старше 60 лет частота регистрации инфицирования SARS-Cov-2 составила 27,1%. Данные показатели, учитывая длительную социальную разобщенность этой категории граждан при введении ограничительных мероприятий, могут быть объяснены клинически выраженным течением заболевания, послужившим причиной обращения за медицинской помощью. Удельный вес детей до 18 лет составил 8,3 \%, и, учитывая раннее разобщение организованных детских коллективов, свидетельствует о вероятном их заражении в семейных очагах.

Установлен неравномерный характер распределения заболеваемости новой коронавирусной инфекцией по территории. Наибольшее количество 
больных выявлено в г. Волгограде (41,3 \%) и г. Волжском (7,6 \%) (рис. 2). В целом на городских жителей пришлось 69,5 \% случаев заражений. При сравнительном анализе распределения заболеваемости среди городского и сельского населения по возрастам, установлено, что количество заболевших сельских жителей превалирует в группе 50-59 лет. Это наблюдение требует дальнейшего детального изучения.

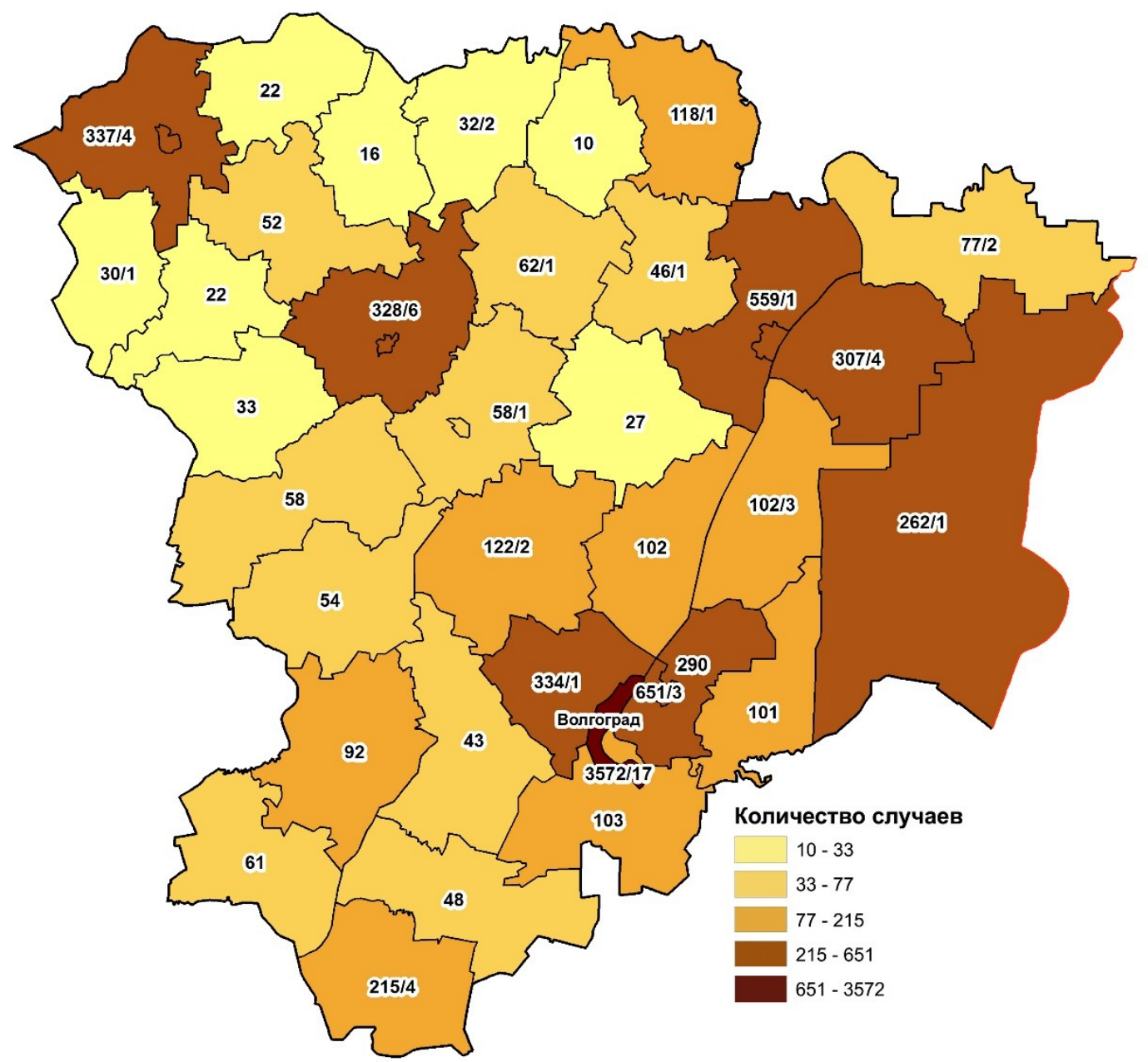

Рис. 2. Дифференциация территории Волгоградской области по уровню заболеваемости COVID-19 (по данным на 22.07.2020 г.)

Примечание: через дробь указано количество летальных случаев.

В ходе эпидемиологических расследований показано, что в 40,4 \% случаев заражение происходит в семейных очагах, 31,5 \% - от неустановленного источника, 11,1 \% - в медицинских организациях, 17 \% других очагах. Высокий процент случаев заражения от неустановленного 
источника, по всей вероятности, связан с заражениями в результате контактов с больными бессимптомной формой. Это подтверждают данные зарубежных публикаций, указывающие, что больные бессимптомной формой являются источниками инфекции в 6-41% случаях [9].

Анализ особенностей клинических проявлений заболевания позволяет установить, что бессимптомные и легкие формы течения встречаются в примерно равном соотношении (35,4 и 34,5 \% соответственно). Доля лабораторно подтвержденных случаев COVID-19 без клинических проявлений в Волгоградской области ниже, чем в целом по России (от 40 \% и выше) [3]. В течение периода стабильного снижения общей заболеваемости отмечается тенденция сокращения количества бессимптомных форм. Самый низкий показатель выявления бессимптомного течения COVID-19 отмечен на 28-й неделе (1,6 \% от общего количества больных). Данное наблюдение может быть объяснено уменьшением общего числа очагов заболеваемости, характеризующихся высокой частотой случаев без развития симптомов, и не связано с объемом проводимого тестирования, показатели которого в указанный период оставались стабильными.

Среднетяжелое течение заболевания наблюдается $\quad$ у 27,7 \% инфицированных SARS-Cov-2, тяжелое - 2,4 \%. Увеличение доли тяжелого течения заболевания зафиксировано однократно на 21 неделе (3,5 \% от общего числа случаев), не сопровождавшееся ростом числа летальных исходов. Следует отметить, что доля тяжелых форм COVID-19 среди заболевших в Волгоградской области ниже, чем по Российской Федерации (4,8 \%) [4] и миру (4,7-5 \%) [7, 11]. На уменьшение доли тяжелого течения коронавирусной инфекции могло повлиять заблаговременное проведение превентивных мероприятий в отношении наиболее угрожаемых по развитию данной формы контингентов риска (пенсионеры, лица, страдающие хроническими заболеваниями), а также наличие достаточных мощностей (кадровый ресурс, коечный фонд, средства лечения) медицинских организаций и своевременная госпитализация больных. 
Общее количество летальных исходов по данным на 24.07.2020 г. составило 57 случаев (летальность - 0,66 \%, показатель летальности - 2,28 на 100 тыс. населения). При распределении летальных случаев по возрастным группам установлено, что 81,1 \% смертей отмечено в группе лиц 50 лет и старше. Летальность в возрастной группе от 50 лет и старше составила 0,9 \%, в группе 65 лет и старше - 1,5 \%, что ниже общероссийских (1,2 и 4,8 \% соответственно) [3] и мировых показателей $(3,4$ \% и более) $[7,8,11]$. На возраст 30-49 лет пришлось 18,9 \% летальных исходов. Случаев смерти в возрасте до 30 лет не зарегистрировано. Смертельные исходы среди мужчин регистрировались в 1,5 раза чаще, чем среди женщин.

Все пациенты с летальным исходом имели в анамнезе одно или несколько сопутствующих хронических заболеваний. В подавляющем большинстве случаев летальные исходы от COVID-19 были ассоциированы с заболеваниями сердечно-сосудистой системы (62,3 \%) и сочетанной патологией эндокринной и сердечно-сосудистой систем (22,6 \%). По данным зарубежных публикаций, пациенты, умершие от COVID-19, чаще страдали хроническими заболеваниями сердечно-сосудистой системы, сахарным диабетом, ожирением, хроническими респираторными заболеваниями и злокачественными новообразованиями [6, 8, 11]. Важность своевременного обращения за медицинской помощью подчеркивают данные о регистрации 68 \% летальных исходов среди пациентов, обратившихся в медицинское учреждение на третий и более поздний день от начала заболевания.

С момента начала регистрации заболеваемости в организованных коллективах выявлено 27 очагов с общим число пострадавших 1119 человек (13,4 \% от общего числа больных), в том числе в медицинских организациях - 16 очагов (421 случай), в интернатных и социальных учреждениях - 4 очага (370 случаев). 


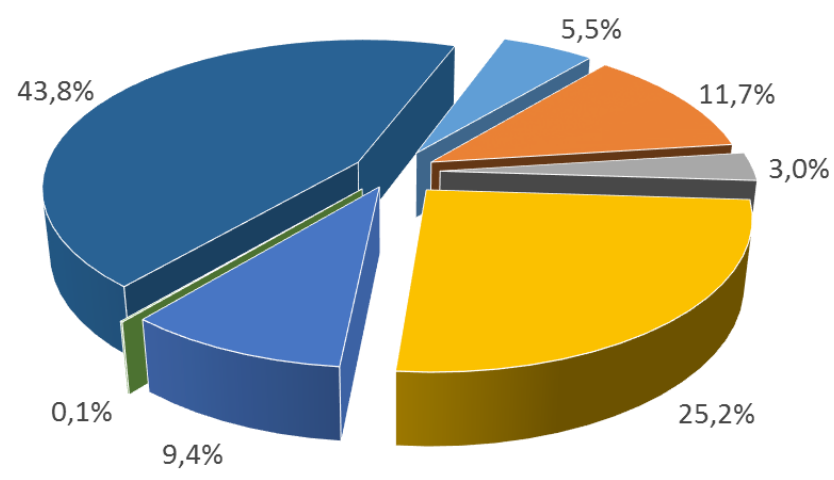

- Воспитанники/учащиеся

- Рабочие

- Служащие

П Пенсионеры

- Работники медицинских организаций

- Представители силовых структур

- Другое

Рис. 3. Распределение заболеваемости COVID-19 по социальным группам

В социальной структуре заболевших наибольшую долю составляют пенсионеры, рабочие и работники медицинских организаций (рис. 3). За анализируемый период наблюдения (с 22.05 по 22.07) отметилось незначительное увеличение доли пенсионеров в общей структуре заболеваемости с 20,4 \% до 25,2 \%, что, вероятно, связано с повышением их социальной активности (выезд на дачу, к месту работы) в результате смягчения ограничительных мер. Среди контингента рабочих произошло снижение заболеваемости, доля рабочих составила 11,7 \% против 22,6 \%. Данное наблюдение может быть объяснено снижением трудовой активности данной социальной группы ввиду продолжающейся реализации комплекса профилактических мероприятий и наступлением сезона отпусков. Частота выявления новой коронавирусной инфекции в группе воспитанники/учащиеся в течение последних месяцев оставалась стабильной (в среднем 5,6 \%) в связи с сохранением разобщения организованных детских коллективов.

В то же время отмечается рост числа заболевших среди медицинских работников и, соответственно, возрастает их доля в общей структуре заболеваемости - с 6,1 \% до 9,4 \%. Это определяет необходимость строгого 
соблюдения требований биологической безопасности при осуществлении профессиональной деятельности как в специализированных медицинских учреждениях, так и общей медицинской сети. По данным зарубежных публикаций, уровень инфицирования среди медицинского персонала варьирует в широких пределах от 2,1 до 29 \% [6, 8, 11].

В целом анализ эпидемиологической ситуации по COVID-19 в Волгоградской области свидетельствует о стабильном снижении заболеваемости новой коронавирусной инфекций, проявляющейся сокращением общей и групповой заболеваемости, количества семейных очагов инфекции, числа госпитализированных пациентов. Оценка эпидемического процесса COVID-19 среди различных групп населения (пол, возраст, социальный статус) и особенностей клинической форм заболевания демонстрирует сходный характер проявлений, зарегистрированный в Российский Федерации и в мире.

Список литературы:

1. Вступительное слово Генерального директора на пресс брифинге по COVID-19 11 марта 2020 г. URL: https://www.who.int/ru/dg/speeches/detail/who-director-general-sopening-remarks-at-the-media-briefing-on-covid-19---11-march-2020 (дата обращения: 20.07.2020 г.).

2. Заявление по итогам второго совещания Комитета по чрезвычайной ситуации в соответствии с Международными медико-санитарными правилами, в связи со вспышкой заболевания, вызванного новым коронавирусом 2019 г. (nCoV) URL: https://www.who.int/ru/news-room/detail/30-01-2020-statement-on-the-second-meeting-of-theinternational-health-regulations-(2005)-emergency-committee-regarding-the-outbreak-of-novelcoronavirus-(2019-ncov) (дата обращения: 19.07.2020 г.).

3. Кутырев В.В., Попова А.Ю., Смоленский В.Ю., Ежлова Е.Б., Демина Ю.В., Сафронов В.А., Карнаухов И.Г., Иванова А.В., Щербакова С.А. Эпидемиологические особенности новой коронавирусной инфекции (COVID-19). Сообщение 2: особенности течения эпидемического процесса COVID-19 во взаимосвязи с проводимыми противоэпидемическими мероприятиями в мире и Российской Федерации. Проблемы особо опасных инфекций. 2020;(2):6-12. https://doi.org/10.21055/0370-1069-2020-2-6-12. 
4. Пшеничная Н.Ю. 2020. Эпидемический процесс COVID-19 в Российской Федерации: промежуточные итоги. Сообщение 1. covid19-preprints.microbe.ru. https://doi.org/10.21055/preprints-3111725.

5. Adhikari S.P., Meng S., Wu Y.J. et al. Epidemiology, causes, clinical manifestation and diagnosis, prevention and control of coronavirus disease (COVID-19) during the early outbreak period: a scoping review. Infect Dis Poverty. 2020;9(1):29. Published 2020 Mar 17. doi:10.1186/s40249-020-00646-x.

6. Epidemiology of COVID-19. European Centre for Disease Prevention and Control. URL: https://www.ecdc.europa.eu/en/covid-19/latest-evidence/epidemiology (дата обращения: 22.07.2020 г.).

7. Epidemiology Working Group for NCIP Epidemic Response, Chinese Center for Disease Control and Prevention. The epidemiological characteristics of an outbreak of 2019 novel coronavirus diseases (COVID-19) in China. Zhonghua Liu Xing Bing Xue Za Zhi. 2020;41(2):145-151. doi:10.3760/cma.j.issn.0254-6450.2020.02.003.

8. Ge H., Wang X., Yuan X., et al. The epidemiology and clinical information about COVID-19. Eur J Clin Microbiol Infect Dis. 2020;39(6):1011-1019. doi:10.1007/s10096-02003874-z.

9. Transmission of COVID-19. European Centre for Disease Prevention and Control. URL: https://www.ecdc.europa.eu/en/covid-19/latest-evidence/transmission (дата обращения: 22.07.2020 г.).

10. WHO Statement regarding cluster of pneumonia cases in Wuhan, China. URL: https://www.who.int/china/news/detail/09-01-2020-who-statement-regarding-cluster-ofpneumonia-cases-in-wuhan-china (дата обращения: 19.07.2020 г.).

11. Wu Z., McGoogan J.M. Characteristics of and Important Lessons From the Coronavirus Disease 2019 (COVID-19) Outbreak in China: Summary of a Report of 72314 Cases From the Chinese Center for Disease Control and Prevention. JAMA. 2020 Feb 24. 